Article

\title{
Sub-Harmonic Response Analysis of Nonlinear Dynamic Behaviors Induced by Piecewise-Type Nonlinearities in a Torsional Vibratory System
}

\author{
Jong-Yun Yoon ${ }^{1}$ and Byeongil Kim ${ }^{2, *(D)}$ \\ 1 Department of Mechatronics Engineering, Incheon National University, (Songdo-dong) 119 Academy-ro, \\ Yeonsu-gu, Incheon 22012, Korea; yoon3932@inu.ac.kr \\ 2 School of Mechanical Engineering, Yeungnam University, (Dae-dong) 280 Daehak-ro, \\ Gyeongsan-si 38541, Korea \\ * Correspondence: bikim@yu.ac.kr; Tel.: +82-53-810-2447
}

check for updates

Citation: Yoon, J.-Y.; Kim, B.

Sub-Harmonic Response Analysis of Nonlinear Dynamic Behaviors Induced by Piecewise-Type

Nonlinearities in a Torsional

Vibratory System. Appl. Sci. 2022, 12, 1845. https://doi.org/10.3390/ app12041845

Academic Editors: Alberto Doria and Alessandro Gasparetto

Received: 20 December 2021

Accepted: 9 February 2022

Published: 10 February 2022

Publisher's Note: MDPI stays neutral with regard to jurisdictional claims in published maps and institutional affiliations.

Copyright: (C) 2022 by the authors. Licensee MDPI, Basel, Switzerland. This article is an open access article distributed under the terms and conditions of the Creative Commons Attribution (CC BY) license (https:// creativecommons.org/licenses/by/ $4.0 /)$.

\begin{abstract}
Piecewise-type nonlinearities, such as clutch dampers in a torsional system, induce complex nonlinear dynamic behaviors that resemble super- and sub-harmonic responses. This study focuses on investigating the sub-harmonic responses induced by piecewise-type nonlinearities in the middle of various dynamic behaviors in a torsional vibratory system. To examine the dynamic characteristics in a sub-harmonic regime, the harmonic balance method (HBM) was implemented. Its results were compared with the numerical simulation (NS). To reveal the sub-harmonic responses, the input conditions of the HBM were modified with a small number of input values. In addition, bifurcation diagrams were numerically determined and projected onto stable and unstable solutions of the HBM to examine the effective dynamic behaviors within the unstable regimes. The results of the HBM with the modified input conditions reveal the sub-harmonic effects well, and the comparisons of bifurcation diagrams under unstable conditions lead to an understanding of the complex dynamic behaviors. Overall, this study suggests the first analytical technique to determine the sub-harmonic responses with the HBM, and second investigates the complex dynamic behaviors in a practical vibratory system by considering the bifurcations in the unstable regimes.
\end{abstract}

Keywords: harmonic balance method; piecewise-type nonlinearities; sub-harmonic response; bifurcation; multi-staged clutch damper; torsional system

\section{Introduction}

Piecewise-type nonlinearities such as multi-staged clutch dampers used in a practical torsional system induce highly complex dynamic responses. In the middle of these nonlinear dynamic behaviors, sub-harmonic responses are relatively difficult to detect by employing the harmonic balance method (HBM), the basic matrix of which is constructed by the integer-based, incremental formulations [1-19]. In addition, various modifications have been implemented to calculate sub-harmonic responses. For example, nonlinear frequency response calculations of a torsional system with clearance-type nonlinearity have been developed by employing a multiterm HBM $[1,2,5,8,10,12]$. Our study suggested adapting the relevant time period for the sub-harmonic frequency values. Duan et al. suggested an excitation perturbation method to investigate the sub-harmonic resonance [10]. To capture the sub-harmonic effects, the authors modified the input conditions. For example, the relevant sub-harmonic input terms were artificially included, which triggered relevant subharmonic responses. In addition, various prior studies have discussed nonlinear problems by employing the HBM with respect to super- and sub-harmonic responses $[2,7,10,12]$.

Despite the successful determination of the sub-harmonic responses, the stability conditions of the HBM often cannot adequately explain the practical dynamic conditions. 
To understand the dynamic behaviors in complex stable and unstable conditions, bifurcation diagrams have been presented based on time-domain solutions. With regard to bifurcations, various approaches and results have been discussed. For instance, Al-shyyab and Kahraman investigated sub-harmonic and chaotic motions in a multi-mesh gear train by using a nonlinear time-varying dynamic model [5]. Detroux et al. examined bifurcation phenomena in a codimension-2 parameter space by using the HBM [14]. Xie et al. suggested the original extended system model for the detection and tracking of bifurcations by using the HBM with an arc-length continuation technique [15].

To the best of our knowledge based on the HBM and its relevant techniques, this study investigates the dynamic characteristics that occur in the sub-harmonic regimes, which are primarily concerned with a practical multi-staged clutch dampers that are employed in the conventional vehicle system. Thus, there are two specific objectives of this study. The first objective is to investigate the sub-harmonic regime which is just determined as unstable conditions based on the HBM. To capture the sub-harmonic resonances, the fictitious subharmonic input conditions will be employed numerically, which will advance the prior study [2]. The second objective is to examine the complex dynamic characteristics caused by a physical multi-staged clutch dampers by investigating the bifurcation diagrams that generally occur in the sub-harmonic unstable regimes. This will result in an increased understanding regarding the practical dynamic motions in a vehicle driveline system. In addition, this study focuses on one specific multi-staged clutch damper model in a torsional system with a single-degree-of-freedom (1 DOF) by limiting the sub-harmonic resonance areas around $\omega=1$. In addition, Broader range of dynamic responses and their analysis of the bifurcation characteristics in the same system as described in Figure 1 can be referred [20].

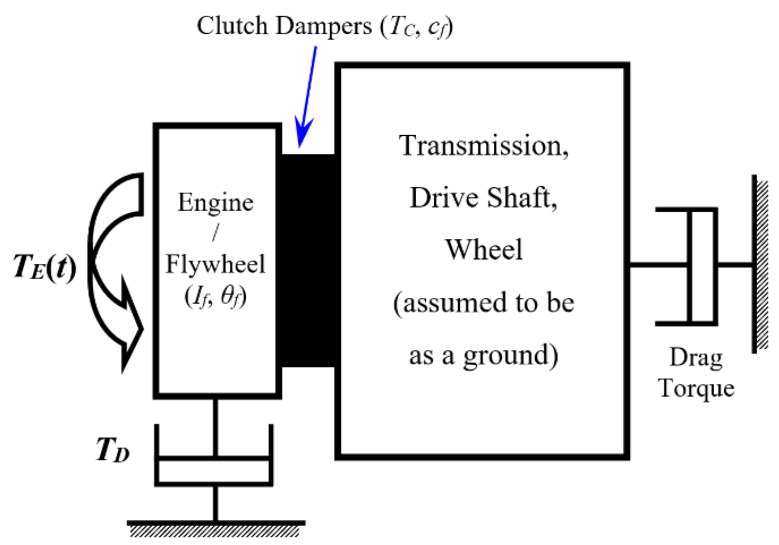

(a)

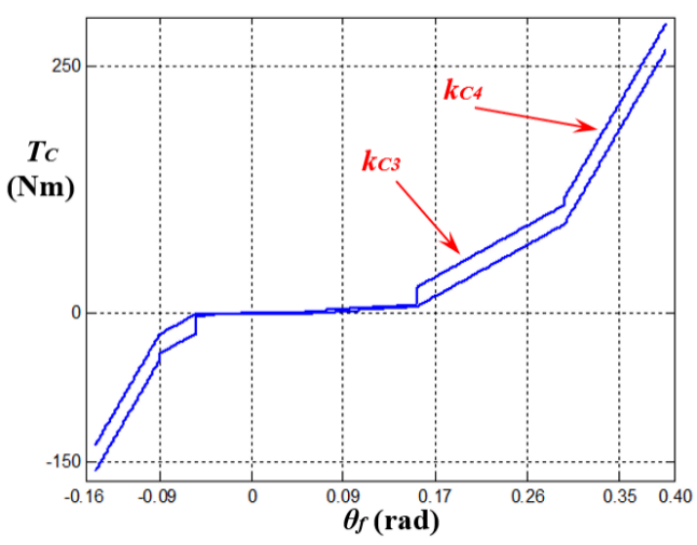

(b)

Figure 1. A single-degree-of-freedom system with piecewise type nonlinearities: (a) a nonlinear torsional system model with $1 \mathrm{DOF}$; $(\mathbf{b})$ Torque $T_{C}\left(\delta_{1}\right)$ profile for a multi-staged clutch damper $[1,2]$.

\section{Problem Formulation with Multi-Staged Clutch Dampers}

Figure 1a shows the nonlinear vibratory system with 1 DOF affected by piecewise-type nonlinearities based on multi-staged clutch dampers. The nonlinear model for piecewisetype nonlinearities is depicted in Figure $1 \mathrm{~b}$.

To investigate the dynamic characteristics, the employed parameters for the torsional system shown in Figure 1a are as follows [1,2]: inertia of flywheel, $I_{f}=1.38 \times 10^{-1} \mathrm{~kg} \cdot \mathrm{m}^{2}$; viscous damping, $c_{f}=1.59 \mathrm{~N} \cdot \mathrm{m} \cdot \mathrm{s} / \mathrm{rad}$. Here, the employed natural frequency $\omega_{n}$ is $115.6(\mathrm{rad} / \mathrm{s})$ from $I_{f}$ and 4 th stage of stiffness value listed in Table 1. In addition, $\theta_{f}$ is the absolute displacement of the flywheel (subscript $f$ ), as indicated in Figure 1a. The profiles of the clutch torque $T_{C}$ are listed in Table 1 , where $k_{C i}$ and $H_{i}(i=1,2,3$, and 4) are the torsional stiffness and hysteresis at the $i^{\text {th }}$ stage, respectively, and $\phi_{p i}$ (or $\phi_{n i}$ ) is the transition angle at the positive (or negative) side [1,2]. Figure $1 \mathrm{~b}$ illustrates the $3 \mathrm{rd}$ and 
4th stages of stiffness and hysteresis areas. Based on the 1 DOF shown in Figure 1a, the equation of motion is derived as follows:

$$
I_{f} \ddot{\theta}_{f}(t)+c \dot{\theta}_{f}(t)+f_{n}\left(\theta_{f}, \dot{\theta}_{f}\right)=T_{E}(t)-T_{D}
$$

Table 1. Properties of the real-life multi-staged clutch damper [1,2].

\begin{tabular}{ccc}
\hline Property & Stage & Value \\
\hline Torsional stiffness, $k_{C i}$ & 1 & 10.1 \\
(linearized in a piecewise manner) & 2 & 61.8 \\
$(\mathrm{Nm} /$ rad $)$ & 3 & 595.8 \\
& 4 & 1838.0 \\
\hline & 1 & 0.98 \\
Hysteresis, $H_{i}(\mathrm{Nm})$ & 2 & 1.96 \\
& 3 & 19.6 \\
& 4 & 26.5 \\
\hline & 1 & 0.05 \\
Transition angle at positive side & 2 & 0.16 \\
$\left(\theta_{f}>0\right), \phi_{p i}(\mathrm{rad})$ & 3 & 0.30 \\
& 4 & 0.39 \\
\hline & 1 & -0.04 \\
Transition angle at negative side & 2 & -0.05 \\
$\left(\theta_{f}<0\right), \phi_{n i}(\mathrm{rad})$ & 3 & -0.09 \\
& 4 & -0.15 \\
\hline
\end{tabular}

Here, $f_{n}\left(\theta_{f}, \dot{\theta}_{f}\right)$ is a nonlinear function that describes the nonlinear clutch forces affected by piecewise-type nonlinearities. $T_{E}(t)$ and $T_{D}$ are the sinusoidal input and drag torque, respectively.

In general, the input torque can be calculated using the Fourier coefficients based on the measured data, as follows:

$$
T_{E}(t)=T_{m}+\sum_{i=1}^{N_{\max }} T_{p i} \cos \left(i \omega_{p} t+\varphi_{p i}\right) .
$$

Here, $T_{m}$ and $T_{p i}$ are the mean and alternating parts of the input torque, respectively; $\omega_{p}$ and $\varphi_{p i}$ are the excitation frequency and phase angle, respectively; and $N_{\max }$ is the maximum number of harmonics correlated with the harmonic index of the HBM. The input torque profiles employed are listed in Table 2. In this study, the drag torque is assumed as $T_{D}=T_{m}$ under steady-state conditions.

Table 2. List of the input torque profiles by assuming the WOT condition on the vehicle $[1,2]$.

\begin{tabular}{cccc}
\hline \multicolumn{2}{c}{ Torque Component } \\
\cline { 1 - 2 } & Magnitude (Nm) & Phase (rad) \\
\hline$T_{M}$ & $T_{p 1}$ & -1.93 \\
\hline 168.9 & 251.5 & \\
\hline
\end{tabular}

In addition to the input conditions, $f_{n}\left(\theta_{f}, \dot{\theta}_{f}\right)$ as illustrated in Figure 1, is the main nonlinearity affected by multiple components, such as piecewise linear stiffnesses, hysteresis, and pre-load. Here, $T_{S P r}$ is the total clutch torque induced by the pre-load, $T_{P r 1}$ (or $T_{P r 2}$ ) is the positive (or negative) torque induced by the pre-load, and $\phi_{P r}$ is the angle located at the pre-load. Based on prior studies, the nonlinear function $f_{n}\left(\theta_{f}, \dot{\theta}_{f}\right)$ (or $\left.T_{C}\right)$ is derived as 
follows [1,2]: First, the clutch torque induced by the stiffness $T_{S}\left(\theta_{f}\right)$ was defined using the smoothing factor $\sigma_{C}$.

$$
\begin{gathered}
T_{S}\left(\theta_{f}\right)=k_{C 1} \theta_{f}+\frac{1}{2} \sum_{i=2}^{N}\left(k_{C(i)}-k_{C(i-1)}\right)\left(T_{s p(i-1)}-T_{s n(i-1)}\right), \\
T_{s p(i)}=\left(\theta_{f}-\phi_{p(i)}\right)\left[\tanh \left\{\sigma_{C}\left(\theta_{f}-\phi_{p(i)}\right)\right\}+1\right] \\
T_{s n(i)}=\left(\theta_{f}+\phi_{n(i)}\right)\left[\tanh \left\{\sigma_{C}\left(\theta_{f}+\phi_{n(i)}\right)\right\}-1\right] .
\end{gathered}
$$

Here, $k_{C(N)}$ (or $k_{C(i)}$ ) is the $N^{\text {th }}$ (or $i^{\text {th }}$ ) stage of the clutch stiffness (with subscript $N$ or i), $T_{s p(i)}\left(\right.$ or $\left.T_{s n(i)}\right)$ is the positive (or negative) direction of the clutch torque induced by the stiffness at the $i^{\text {th }}$ stage (with subscript $p$ or $n$ ), and $\phi_{p(i)}\left(\right.$ or $\left.-\phi_{n(i)}\right)$ is the $i^{\text {th }}$ transition angle of the positive (or negative) side [3]. Second, $T_{H}$ induced by hysteresis was derived using the smoothing factor $\sigma_{H}[1,2]$.

$$
\begin{gathered}
T_{H}\left(\theta_{f}, \dot{\theta}_{f}\right)=\frac{H_{(N)}}{2} \tanh \left(\sigma_{H} \dot{\theta}_{f}\right)+\sum_{i=2}^{N}\left(\frac{H_{(i)}}{4}-\frac{H_{(i-1)}}{4}\right)\left[T_{H p(i-1)}+T_{H n(i-1)}\right], \\
T_{H p(i)}=\tanh \left\{\sigma_{C}\left(\theta_{f}-\phi_{p(i)}\right)\right\}\left[1+\tanh \left(\sigma_{H} \dot{\theta}_{f}\right)\right], \\
T_{H n(i)}=\tanh \left\{\sigma_{C}\left(\theta_{f}+\phi_{n(i)}\right)\right\}\left[1-\tanh \left(\sigma_{H} \dot{\theta}_{f}\right)\right] .
\end{gathered}
$$

Here, $H_{N}\left(\right.$ or $\left.H_{(i)}\right)$ is the $N^{\text {th }}$ (or $i^{\text {th }}$ ) stage of hysteresis (with subscript $N$ or $i$ ), and $T_{H p(i)}$ (or $T_{H n(i)}$ ) is the positive (or negative) side of the clutch torque induced by hysteresis at the $i^{\text {th }}$ stage (with subscript $p$ or $n$ ). The pre-load $T_{S P r}$ was calculated as a function of $\theta_{1 p r}$.

$$
\begin{gathered}
T_{S P r}\left(\theta_{1 p r}\right)=\frac{1}{2} T_{P r 1}\left[\tanh \left(\sigma_{C} \theta_{1 p r}\right)+1\right]+\frac{1}{2} T_{P r 2}\left[-\tanh \left(\sigma_{C} \theta_{1 p r}\right)+1\right], \\
\theta_{1 p r}=\theta_{f}-\phi_{P r} .
\end{gathered}
$$

Overall, the total clutch torque is estimated by the summation of, $T_{S}\left(\theta_{f}\right), T_{H}\left(\theta_{f}, \dot{\theta}_{f}\right)$, and $T_{S P r}\left(\theta_{1 p r}\right)$ from Equations (3)-(5), as follows:

$$
f_{n}\left(\theta_{f}, \dot{\theta}_{f}\right)=T_{C}\left(\theta_{1 p r}, \dot{\theta}_{1 p r}\right)=T_{S}\left(\theta_{1 p r}\right)+T_{H}\left(\theta_{1 p r}, \dot{\theta}_{1 p r}\right)+T_{S P r}\left(\theta_{1 p r}\right) .
$$

The employed values for $\sigma_{C}$ and $\sigma_{H}$ are $1 \times 10^{3}$ and 0.1 , respectively.

\section{HBM with Modified Input Conditions}

\subsection{Basic Formulation of the HBM}

From Equation (1), the Galerkin scheme of the governing equations is expressed as follows [2,12]:

$$
-\omega^{2} m \underline{\underline{\mathbf{H P}^{\prime \prime}}} \underline{\theta_{c}}+\omega c \underline{\underline{\mathbf{H P}}} \underline{\theta}^{\prime}+\underline{f_{n}}\left(\underline{\theta_{f}}, \underline{\theta_{f}}\right)-\underline{F_{E}}(t)=\underline{0} .
$$

Here, its corresponding formulas are defined as follows:

$$
\begin{aligned}
& \underline{\theta_{f}}(t)=\underline{\underline{\mathbf{H}}} \underline{\underline{\theta_{c}}}, \\
& \underline{\theta_{f}}(t)=\left[\begin{array}{lllll}
\theta_{f}\left(t_{0}\right) & \theta_{f}\left(t_{1}\right) & \cdots & \theta_{f}\left(t_{m-2}\right) & \theta_{f}\left(t_{m-1}\right)
\end{array}\right]^{T}, \\
& \underline{\theta_{c}}=\left[\begin{array}{lllllllll}
\theta_{m} & \theta_{a(1)} & \theta_{b(1)} & \cdots & \theta_{a(k)} & \theta_{b(k)} & \cdots & \theta_{a\left(\eta N_{\max }\right)} & \theta_{b\left(\eta N_{\max }\right)}
\end{array}\right]^{T} .
\end{aligned}
$$




$$
\begin{aligned}
& \underline{\underline{\mathbf{H}}}=\left[\begin{array}{ccccc}
1 & \cdots & \cos \left(k \Psi_{0}\right) & \sin \left(k \Psi_{0}\right) & \cdots \\
1 & \cdots & \cos \left(k \Psi_{1}\right) & \sin \left(k \Psi_{1}\right) & \cdots \\
& \ddots & & & \ddots \\
1 & \cdots & \cos \left(k \Psi_{N-2}\right) & \sin \left(k \Psi_{N-2}\right) & \cdots \\
1 & \cdots & \cos \left(k \Psi_{N-1}\right) & \sin \left(k \Psi_{N-1}\right) & \cdots
\end{array}\right], \underline{\underline{\mathbf{H}}}^{\prime}=\omega \underline{\underline{\mathbf{H P}}}^{\prime}, \underline{\underline{\mathbf{H}}}^{\prime \prime}=-\omega^{2} \underline{\underline{\mathbf{H P}}}^{\prime \prime}, \\
& \underline{\underline{\mathbf{P}}}^{\prime}=\left[\begin{array}{ccccc}
0 & & & & \\
& \ddots & & & \\
& & {\left[\begin{array}{cc}
0 & k \\
-k & 0
\end{array}\right]} & \\
& & & & \ddots
\end{array}\right], \\
& \underline{\underline{\mathbf{P}}}^{\prime \prime}=\left[\begin{array}{ccccc}
0 & & & & \\
& \ddots & & & \\
& & {\left[\begin{array}{cc}
k^{2} & 0 \\
0 & k^{2}
\end{array}\right]} & \\
& & & & \ddots
\end{array}\right] .
\end{aligned}
$$
follows:

Likewise, its nonlinear and input functions are expressed using the same schemes as

$$
\begin{aligned}
& \underline{f_{n}}\left(\underline{\theta_{f}}, \underline{\dot{\theta}_{f}}\right)=\underline{\underline{\mathbf{H}}} \underline{f_{n c}} \\
& \underline{F_{E}}(t)=\underline{\underline{\mathbf{H}}} F_{E \mathcal{c}}, \\
& \underline{f_{n c}}=\left[\begin{array}{lllllllll}
f_{m} & f_{a(1)} & f_{b(1)} & \cdots & f_{a(k)} & f_{b(k)} & \cdots & f_{a\left(\eta N_{\max }\right)} & f_{b\left(\eta N_{\max }\right)}
\end{array}\right]^{T}, \\
& \underline{F_{E c}}=\left[\begin{array}{lllllllll}
F_{m} & F_{a(1)} & F_{b(1)} & \cdots & F_{a(k)} & F_{b(k)} & \cdots & F_{a\left(\eta N_{\max }\right)} & F_{b\left(\eta N_{\max }\right)}
\end{array}\right]^{T} .
\end{aligned}
$$

Here, let $\omega t=\Psi$ and

non-dimensionalize the time scale and normalize the frequency values. Based on this relationship and by considering the effective time period with the sub-harmonic effect, the relevant time ranges are defined as $0 \leq t<T \rightarrow$, where $0 \leq \Psi<\frac{2 \pi}{\omega_{n}} T=\eta \tau$, where $\eta$ is a sub-harmonic index and $\tau$ is the fundamental frequency. Thus, the index $k$ in Equation (8) is incremented by $k=\omega_{n}, 2 \omega_{n}, 3 \omega_{n} \ldots$ which includes the non-dimensionlized factor $\omega_{n}$. From this relationship, $\dot{\theta}(t)=\frac{d \theta}{d t}=\boldsymbol{\omega} \frac{d \theta}{d \Psi}=\omega \theta^{\prime}$ because $\omega d t=d \Psi$. Likewise, $\ddot{\theta}(t)=\omega^{2} \theta^{\prime \prime}$. The matrices and coefficient vectors are constructed along with the incremental indices $k$ and $\eta$. In addition, the range of the normalized time period should be adapted based on $\eta$. By substituting Equations (8) and (9) into Equation (7), the overall Galerkin scheme of the basic equation is defined as follows.

$$
-\omega^{2} m \underline{\underline{\mathbf{H P}}} \underline{\theta}^{\prime} \underline{\theta_{c}}+\omega c \underline{\underline{\mathbf{H P}}} \underline{\theta}^{\prime}+\underline{\underline{\mathbf{H}}} f_{n c}-\underline{\underline{\mathbf{H}}} F_{E c}=\underline{0} .
$$

Thus,

$$
\begin{gathered}
\underline{\underline{\mathbf{H}} \Psi}=\underline{0}, \\
\underline{\Psi}=-\omega^{2} m \underline{\underline{\mathbf{P}}} \underline{\theta_{c}}+\omega c \underline{\underline{\mathbf{P}}}^{\prime \prime} \underline{\theta_{c}}+\underline{f_{n c}}-\underline{F_{E c}}=\underline{0} .
\end{gathered}
$$

To determine the solutions for $\underline{\theta_{c}}$ in Equations (10) and (11) with their relevant values $\omega$, the Newton-Raphson method is used by considering the condition $\underline{\Psi} \rightarrow \underline{0}$ where $\underline{\Psi}$ is considered as a function of $\underline{\theta}_{\mathcal{c}}$ and $\boldsymbol{\omega}$ such as $\underline{\Psi}\left(\underline{\theta_{\mathcal{C}}}, \boldsymbol{\omega}\right)$. With respect to the implementation of the Newton-Raphson method, the Jacobian matrices for $\underline{\theta_{c}}$ and $\omega$ must be calculated for 
the condition $\underline{\Psi} \cong \underline{0}$. First, the Jacobian matrix $\underline{J_{\mathcal{C}}}$ is considered $\underline{\Psi}$ as a function of $\underline{\theta_{\mathcal{C}}}$, and its equation is as follows:

$$
\underline{J_{c}}=\frac{\partial \underline{\Psi}}{\partial \underline{\theta_{c}}}=-\omega^{2} m \underline{\underline{\mathbf{P}}}^{\prime \prime}+\omega c \underline{\underline{\mathbf{P}}}^{\prime}+\frac{\partial f_{n c}}{\partial \underline{\theta_{c}}} .
$$

Here, to calculate $\frac{\partial f_{n c}}{\partial \underline{\theta_{c}}}$, the derivatives of $\underline{\theta_{f}}(t)$ and $\dot{\theta}_{f}(t)$ must be implemented as follows [2]:

$$
\begin{gathered}
\frac{\partial \underline{f_{n c}}}{\partial \underline{\theta_{c}}}=\underline{\underline{\mathbf{H}}}^{+} \frac{\partial \underline{\partial f_{n}(\underline{\theta}, \underline{\dot{\theta}})}}{\partial \underline{\theta}(t)} \underline{\underline{\mathbf{H}}}+\omega \underline{\underline{\mathbf{H}}}^{+} \frac{\partial \underline{f_{n}(\underline{\theta}, \dot{\theta})}}{\partial \underline{\dot{\theta}}(t)} \underline{\underline{\mathbf{H P}}}^{\prime}, \\
\underline{\underline{\mathbf{H}}}^{+}=\left(\underline{\underline{\mathbf{H}}}^{T} \underline{\underline{\mathbf{H}}}\right)^{-1} \underline{\underline{\mathbf{H}}}^{T} .
\end{gathered}
$$

where $\underline{\underline{\mathbf{H}}}^{+}$is the pseudo-inverse matrix. In addition, $\frac{\partial f_{n}\left(\theta_{f}, \dot{\theta}_{f}\right)}{\partial \underline{\theta_{f}}(t)}$ and $\frac{\partial \underline{f_{n}}\left(\theta_{f}, \dot{\theta}_{f}\right)}{\partial \dot{\theta}_{f}(t)}$ are diagonal matrices defined as follows:

$$
\begin{aligned}
& \frac{\partial \underline{f_{n}}\left(\underline{\theta_{f}}, \dot{\theta}_{f}\right)}{\partial \underline{\theta_{f}}(t)}=\operatorname{diag}\left[\frac{\partial \underline{f_{n}}\left(\underline{\theta_{f}}, \underline{\theta_{f}}\right)}{\partial \underline{\theta_{f}}\left(t_{0}\right)} \frac{\partial \underline{f_{n}}\left(\underline{\theta_{f}}, \underline{\dot{\theta}_{f}}\right)}{\partial \underline{\theta_{f}}\left(t_{1}\right)} \cdots \frac{\partial \underline{f_{n}}\left(\underline{\theta_{f}}, \dot{\theta}_{f}\right)}{\partial \underline{\theta_{f}}\left(t_{m-2}\right)} \frac{\partial \underline{f_{n}}\left(\underline{\theta_{f}}, \dot{\theta}_{f}\right)}{\partial \underline{\theta_{f}}\left(t_{m-1}\right)}\right], \\
& \frac{\partial \underline{f_{n}}\left(\underline{\theta_{f}}, \dot{\theta}_{f}\right)}{\partial \underline{\dot{\theta}_{f}}(t)}=\operatorname{diag}\left[\frac{\partial \underline{f_{n}}\left(\underline{\theta_{f}}, \dot{\theta}_{f}\right)}{\partial \underline{\dot{\theta}_{f}}\left(t_{0}\right)} \frac{\partial \underline{f_{n}}\left(\underline{\theta_{f}}, \underline{\dot{\theta}_{f}}\right)}{\partial \underline{\dot{\theta}_{f}}\left(t_{1}\right)} \cdots \frac{\partial \underline{\underline{f}_{n}}\left(\underline{\theta_{f}}, \dot{\theta}_{f}\right)}{\partial \underline{\dot{\theta}_{f}}\left(t_{m-2}\right)} \frac{\partial \underline{f_{n}}\left(\underline{\theta_{f}}, \underline{\dot{\theta}_{f}}\right)}{\partial \underline{\dot{\theta}_{f}}\left(t_{m-1}\right)}\right] \text {. }
\end{aligned}
$$

Second, the Jacobian matrix $\underline{J_{\omega}}$ by differentiating with respect to $₫$ can be obtained from Equation (11), as follows:

$$
\underline{J_{\omega}}=\frac{\partial \underline{\Psi}}{\partial \omega}=\left(-2 \omega m \underline{\underline{\mathbf{P}}}^{\prime \prime}+c \underline{\underline{\mathbf{P}}}^{\prime}+\underline{\underline{\mathbf{H}}}^{+} \frac{\partial \underline{f_{n}\left(\underline{\theta}_{f}, \dot{\theta}_{f}\right)}}{\underline{\partial \dot{\theta}_{f}(t)}} \underline{\underline{\mathbf{H P}}}\right) \underline{\theta_{c}} .
$$

Overall, the Jacobian matrix with respect to the two parameters $\left(\underline{\theta_{\mathcal{C}}}\right.$ and $\left.\underline{\mathfrak{C}}\right)$ and the augmented vector parameterized by $\omega$ are as follows:

$$
\begin{gathered}
\underline{J}=\left[\begin{array}{ll}
\underline{J_{c}} & \underline{J_{\omega}}
\end{array}\right], \\
\underline{\theta_{a}}=\left[\begin{array}{l}
\frac{\theta_{c}}{\omega}
\end{array}\right] .
\end{gathered}
$$

To obtain the solutions for each step based on the arc-length continuation scheme, the Newton-Raphson technique is conducted as follows:

$$
\underline{\theta}^{(k+1)}=\underline{\theta}^{(k)}-\left[\underline{J}^{+} \underline{\Psi}\left(\underline{\theta}_{c}, \boldsymbol{\omega}\right)\right]^{(k)} .
$$

Here, the pseudo-inverse matrix of the Jacobian is estimated by $\underline{J}^{+}=\left[=\left(\underline{J}^{T} \underline{J}\right)^{-1} \underline{J}^{T}\right]$. In addition, more detailed derivations and techniques for this system in Figure 1 can be found in [2].

\subsection{Initial Results of the Basic HBM and Modification of the Input Conditions}

Figure 2 as initial results shows a comparison of the two results obtained by HBM and NS, where the HBM is conducted with $\eta=2$ and $N_{\max }=12$. To obtain the NS solutions, the modified Runge-Kutta method was employed in this study [1,21]. However, the HBM cannot reveal the sub-harmonic responses even though the solutions of the HBM follow 
the NS results well. This indicates that the sub-harmonic index $\eta$ does not work properly. As for the detailed employment of the NS, it concerned research that can be referred [1,21].

(a)

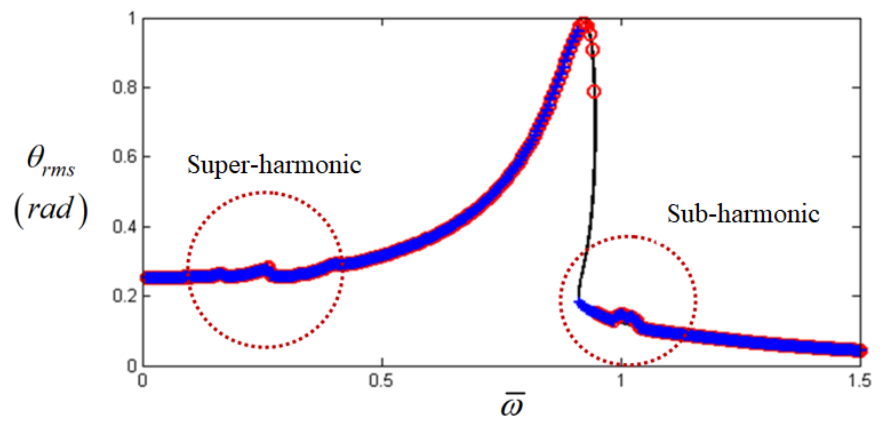

(b)

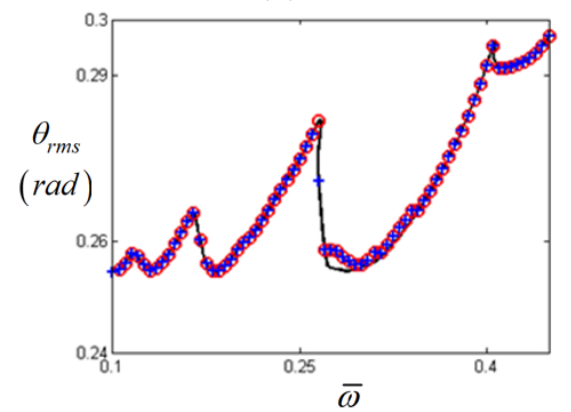

(c)

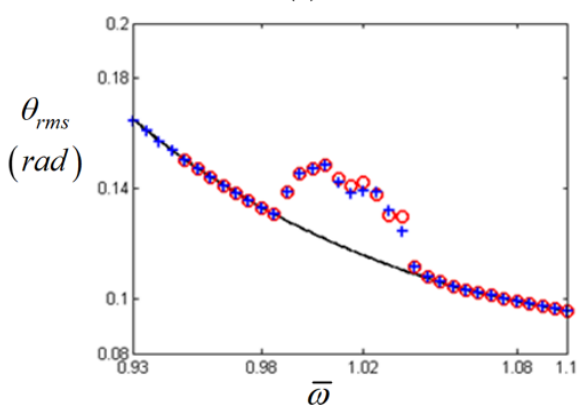

Figure 2. Nonlinear frequency responses with RMS values: (a) comparison of HBM with NS; (b) super-harmonic regions; (c) sub-harmonic region. Key: , HBM result with $\eta=2$ and $N_{\max }=12 ; \mathrm{O}$, NS result with frequency up-sweeping; + , NS result with frequency down-sweeping.

For example, the super-harmonic responses from both the HBM and NS are wellcorrelated, as shown in Figure $2 b$. However, the sub-harmonic regime in Figure 2c shows that the two results of HBM and NS are slightly different because the HBM does not capture the sub-harmonic responses satisfactorily, and its solutions generally reflects the unstable conditions based on Hill's method $[2,12,17]$. However, the NS still projects sub-harmonic responses satisfactorily since the resonance from the calculated results are clearly obtained in Figure 2. Thus, to overcome these discrepancies in the HBM, techniques to trigger the subharmonic effects should be implemented [10]. From Equation (9) and its employed values in Table 2, the valid components of the input torque vector with $\eta=2$ and $N_{\max }=12$ are given as, $F_{m}=168.9, F_{a(2)}=-87.97$, and $F_{b(2)}=235.65$. To trigger the sub-harmonic responses, the components of the input torque pertaining to the sub-harmonic locations, such as $F_{a(1)}$ and, $F_{b(1)}$ can be assigned with small values. In this study, $F_{a(1)}=\varepsilon F_{a(2)}$ and $F_{b(1)}=\varepsilon F_{b(2)}$ were used with $\varepsilon=1 \times 10^{-5}$. With a small range of values multiplied by the fundamental input torque components, the sub-harmonic responses are effectively triggered, as shown in Figure 3. The results in Figure 3a,b successfully capture the sub-harmonic effects with the modified input conditions. Here, regardless of the number of $N_{\max }$ employed, such as $N_{\max }=1$ and $N_{\max }=12$, the sub-harmonic effects are clearly detectable. 

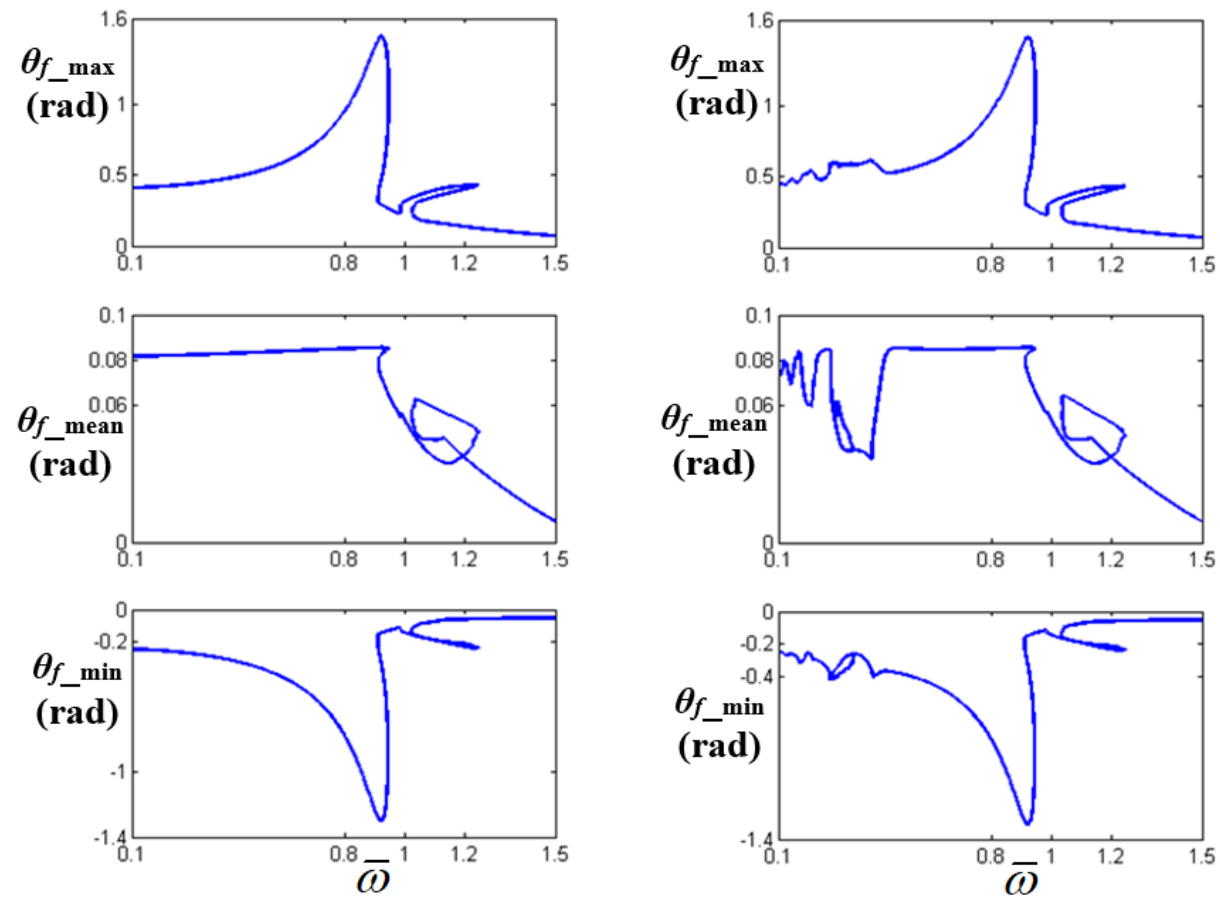

(a)

(b)

Figure 3. Nonlinear frequency responses of HBM reflecting sub-harmonic effects with maximum, mean and minimum values of $\theta_{f}(\mathbf{t})$ : (a) frequency responses of max, mean and min values with $\eta=2$ and $N_{\max }=1 ;(\mathbf{b})$ frequency responses of $\max$, mean and min values with $\eta=2$ and $N_{\max }=12$.

\section{Comparison of the Sub-Harmonic Responses from HBM and NS}

Figure 4 compares the results of the HBM under modified input conditions with the NS solutions. When the sub-harmonic responses are examined carefully, as illustrated in Figure $4 \mathrm{~b}$, the HBM still reflects the discrepancies. As marked with red lines and characters (A) and (B) in Figure $4 \mathrm{~b}$, the calculated results of the HBM at regimes less than (A) or greater than (B) correlate well with those from NS. However, the HBM solutions in the regimes between (A) and (B) are under unstable conditions, and their results do not show good agreement with the NS solutions. To examine the dynamic differences between both methods, the time histories and FFT results can be compared, as shown in Figures 5-7. Figure 5 compares the time histories of the HBM and NS at two different frequencies. Figure 5a shows the time histories based on both HBM and NS at $\omega=111.2\left(\frac{\mathrm{rad}}{\mathrm{s}}\right)$ (or $17.7 \mathrm{~Hz}$ ) that are observed in the regimes below (A) marked in Figure 4b.

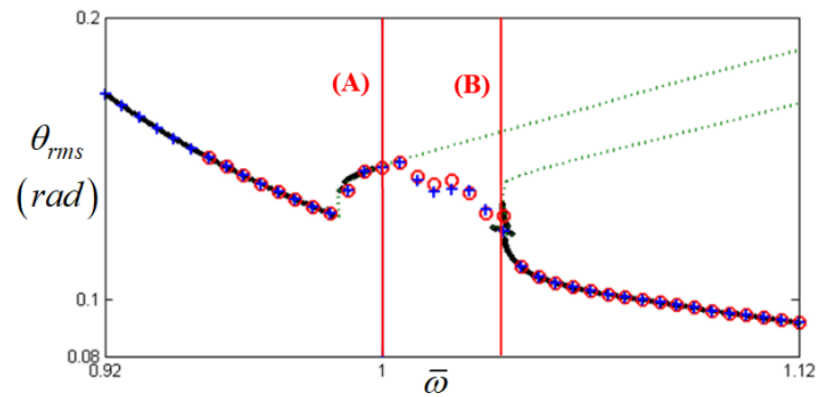

(a)

Figure 4. Cont. 


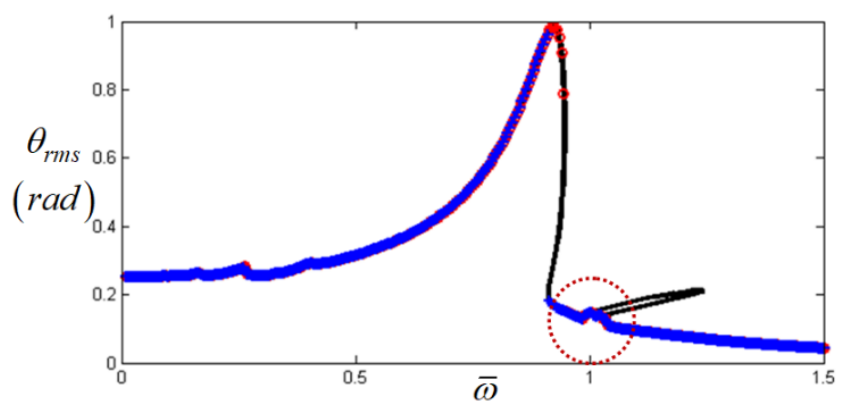

(b)

Figure 4. Comparison of nonlinear frequency responses with RMS values: (a) HBM vs. NS in a whole range of frequency value; (b) HBM vs. NS focused on a sub-harmonic range. Key: —_, stable solution from HBM with $N_{\max }=12$ and $\rho=2 ; \ldots . . . . . "$, unstable solution from HBM with $N_{\max }=12$ and $\rho=2 ; \mathrm{O}$, NS result with frequency up-sweeping; + , NS result with frequency down-sweeping.

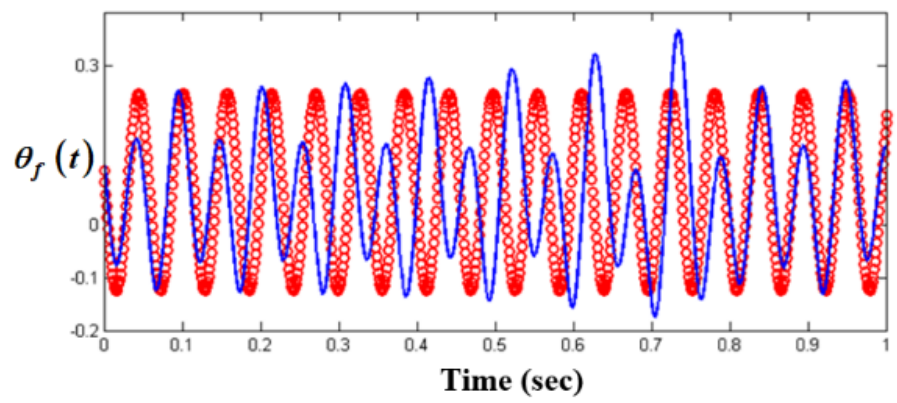

(a)

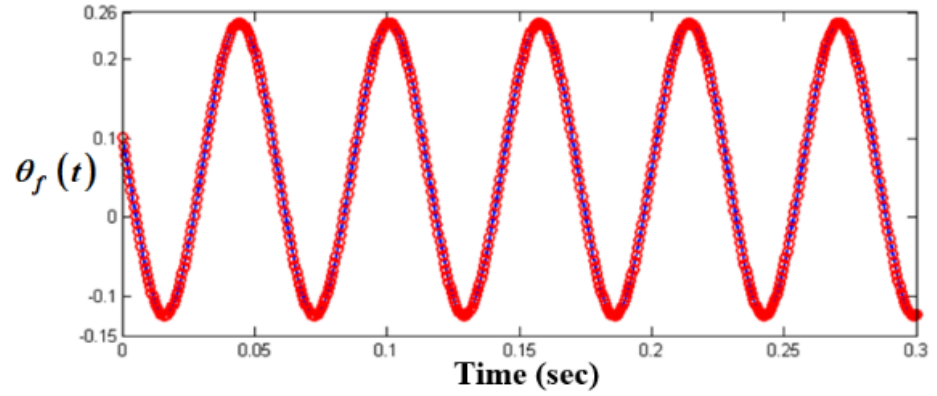

(b)

Figure 5. Comparison of time histories between $\mathrm{HBM}\left(N_{\max }=12\right.$ and $\left.\rho=2\right)$ and NS at different excitation conditions: (a) time histories at $17.7 \mathrm{~Hz}$; (b) time histories at $18.8 \mathrm{~Hz}$. Key: O, HBM; , NS.

As shown in Figure 4, the two calculated time histories coincide with each other. However, the time histories of the HBM at $\omega=118.1$ ( $\frac{\mathrm{rad}}{\mathrm{s}}$ ) (or $18.8 \mathrm{~Hz}$ ) do not show good agreement with the NS results, as shown in Figure 5b, because the HBM generally obtains solutions based on integer-based incremental harmonics. These differences between the HBM and NS are clearly observed in Figures $4 b$ and $5 b$.

In addition, the FFT results reflect the discrepancies between the two methods in unstable regimes. Figure 6a shows that the two FFT results at $\omega=111.2\left(\frac{\mathrm{rad}}{\mathrm{s}}\right)$ (or $17.7 \mathrm{~Hz}$ ) are correlated with each other. However, the FFT results in the unstable regime with $\omega=118.1\left(\frac{\mathrm{rad}}{\mathrm{s}}\right)$ (or $18.8 \mathrm{~Hz}$ ) show discrepancies between the HBM and NS results. The two results still include the sub-harmonic terms clearly identifiable at $\omega=59.1\left(\frac{\mathrm{rad}}{\mathrm{s}}\right)($ or $9.4 \mathrm{~Hz}$ ), as shown in Figure $6 \mathrm{~b}$. Thus, the unstable conditions estimated by the HBM should have a practical meaning that the dynamic behaviors are affected by more complex factors, even 
though the HBM has limitations owing to the integer-based harmonic term simulation. These phenomena result in quasi-harmonic or chaotic responses [22].

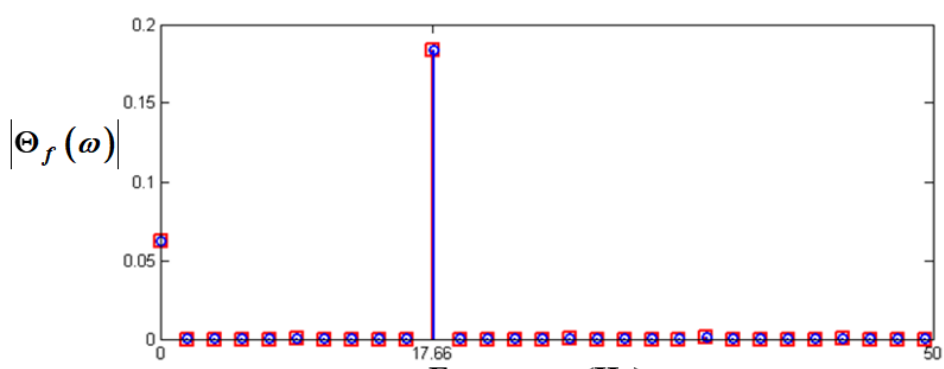

Frequency (Hz)

(a)

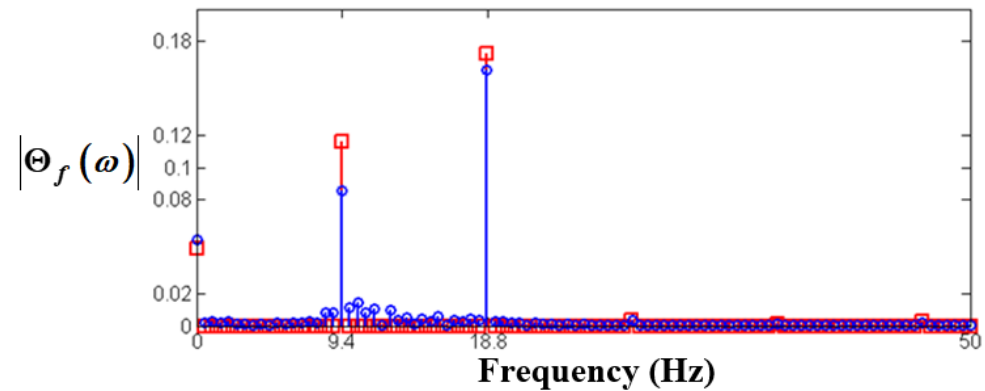

(b)

Figure 6. Comparison of FFT results between HBM $\left(N_{\max }=12\right.$ and $\left.\rho=2\right)$ and NS at different excitation conditions: (a) FFT results at $17.7 \mathrm{~Hz}$; (b) FFT results at $18.8 \mathrm{~Hz}$. Key: $\square$, HBM; O- NS.

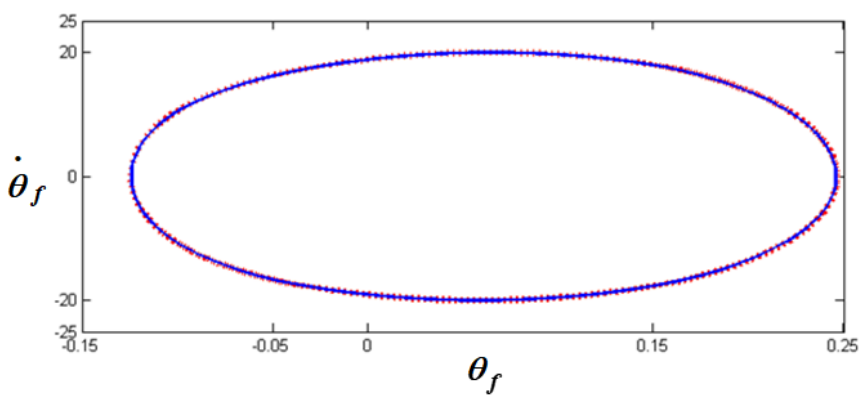

(a)

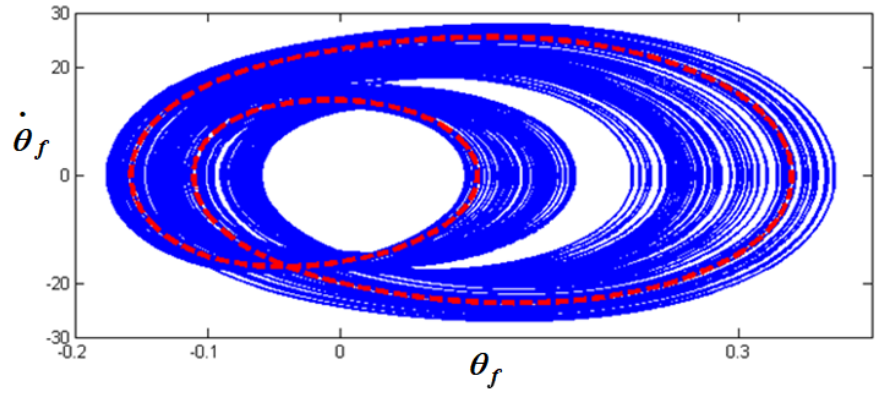

(b)

Figure 7. Comparisons of phase diagrams calculated by HBM $\left(N_{\max }=12\right.$ and $\left.\rho=2\right)$ and NS at different excitation conditions: (a) phase diagrams at $17.7 \mathrm{~Hz}$; (b) phase diagrams at $18.8 \mathrm{~Hz}$. Key: ........, HBM; - NS. 
Figure 7 compares the phase-plane diagrams for two different frequency regimes with $\theta_{f}$ and $\dot{\theta}_{f}$. As seen previously, the phase planes at $\omega=111.2\left(\frac{\mathrm{rad}}{\mathrm{s}}\right)$ (or $\left.17.7 \mathrm{~Hz}\right)$ from the two methods are correlated with each other. In Figure $7 \mathrm{~b}$, both phase-plane diagrams reveal two different effective cycles caused by the fundamental and sub-harmonic responses. In particular, the NS shows more complicated dynamic behaviors that contain many more harmonic terms, which is also confirmed by the number of harmonic components, as shown in Figure 6b.

\section{Dynamic Characteristics with Bifurcation Diagrams in Sub-Harmonic Regimes}

To understand the nonlinear dynamic behaviors, the bifurcation diagrams should be efficient, specifically under the frequency sweeping conditions based on the timedomain solutions. Figures $8 \mathrm{a}$ and $9 \mathrm{a}$ show the maximum $\theta_{r m s}$, mean, and minimum values of $\theta_{f}(t)$ with stable conditions from the HBM. Along with these results, $8 \mathrm{~b}$ and $9 \mathrm{~b}$ reflect the bifurcations that occur in the sub-harmonic regimes. To obtain results from the bifurcation phenomena based on the NS, the solutions for the steady state responses were captured by assuming that the transient responses are completely removed after 100 cycles while frequency sweeping was conducted with $5 \times 10^{-3}$ a frequency step such as $\Delta \omega=5 \times 10^{-3}$. Since the HBM for this study is parameterized by including, $\omega$ as described in Equations (15)-(17), the frequency responses shown in Figures 8a and 9a already include the information of bifurcation areas where unstable conditions are generally observed. Here, to obtain the bifurcation phenomena from the NS, the max, mean, min and rms values for each period are captured repeatedly. In addition, the bifurcations in Figures $8 \mathrm{~b}$ and $9 \mathrm{~b}$ show the common dynamic characteristics in terms of $\theta_{r m s}$, max, mean, and min values of $\theta_{f}(t)$ within the sub-harmonic regimes.

To investigate the dynamic behaviors in the sub-harmonic regimes in more detail, finer temporal steps of NS were conducted and compared with the HBM solutions, as shown in Figures 10 and 11. For instance, bifurcations from NS were obtained with 300 cycles and $\Delta \mathfrak{\omega}=5 \times 10^{-4}$. As shown in Figures 10 and 11, nonlinear dynamic behaviors are clearly observed with respect to the stable and unstable conditions in the sub-harmonic areas. The various bifurcation characteristics are recognized with locations (1), (2), (3), and (4) locations, as illustrated in Figure 10. First, the comparisons between the HBM stable regimes and NS solutions are perfectly matched with each other in the areas prior to (1), and the period doubling occurs at location (1), where the unstable conditions start based on the HBM. Second, another period doubling effect is observed in regime (2). Third, more period doubling and complicated behaviors are clearly seen along with the upper and lower branches of solutions between regimes (2) and (3). Finally, the bifurcations still exist between regimes (3) and (4) even though the HBM indicates stable conditions, and finally the solutions from NS comply with the HBM solutions. The maximum, mean, and minimum values of $\theta_{f}(t)$ also follow the same dynamic characteristics, as shown in Figure 11. Thus, the nonlinear dynamic behaviors can be examined and understood more efficiently by employing both stability and bifurcation analyses. 


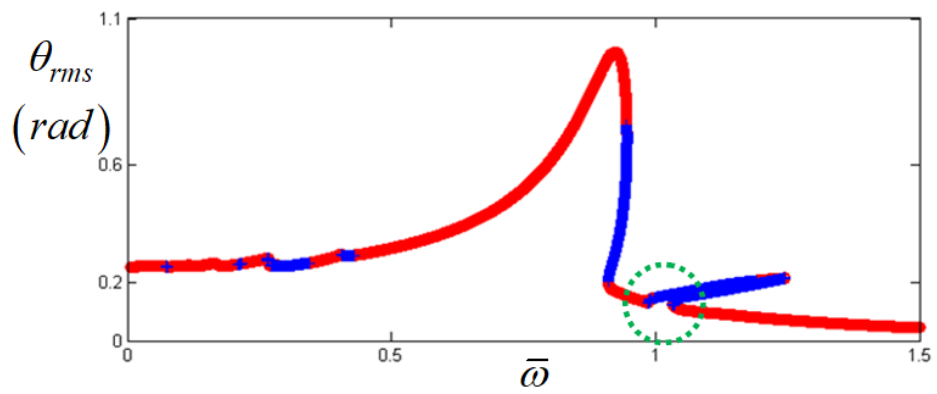

(a)

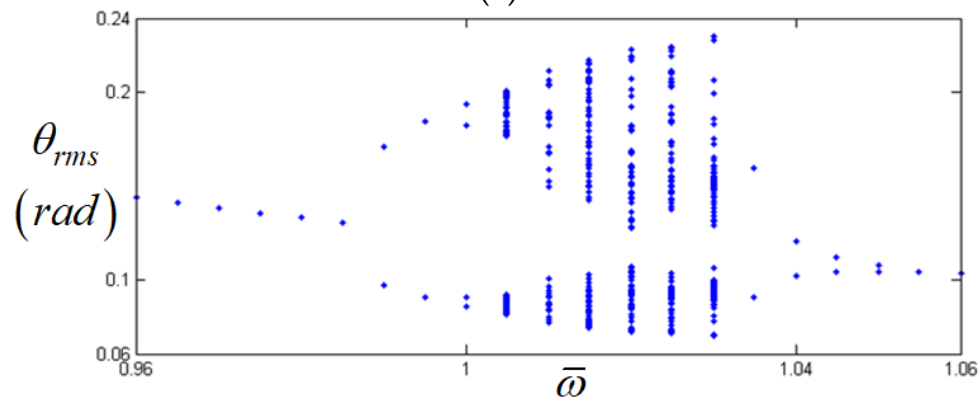

(b)

Figure 8. Stability conditions from HBM with RMS and its bifurcation diagram at the sub-harmonic region: (a) stable and unstable conditions in a frequency response with RMS; (b) bifurcation diagram of the sub-harmonic responses. Key: $\mathbf{O}$, stable solutions of $\mathrm{HBM} ;+$, unstable solutions of HBM; $\bullet$, numerical results of the bifurcation.
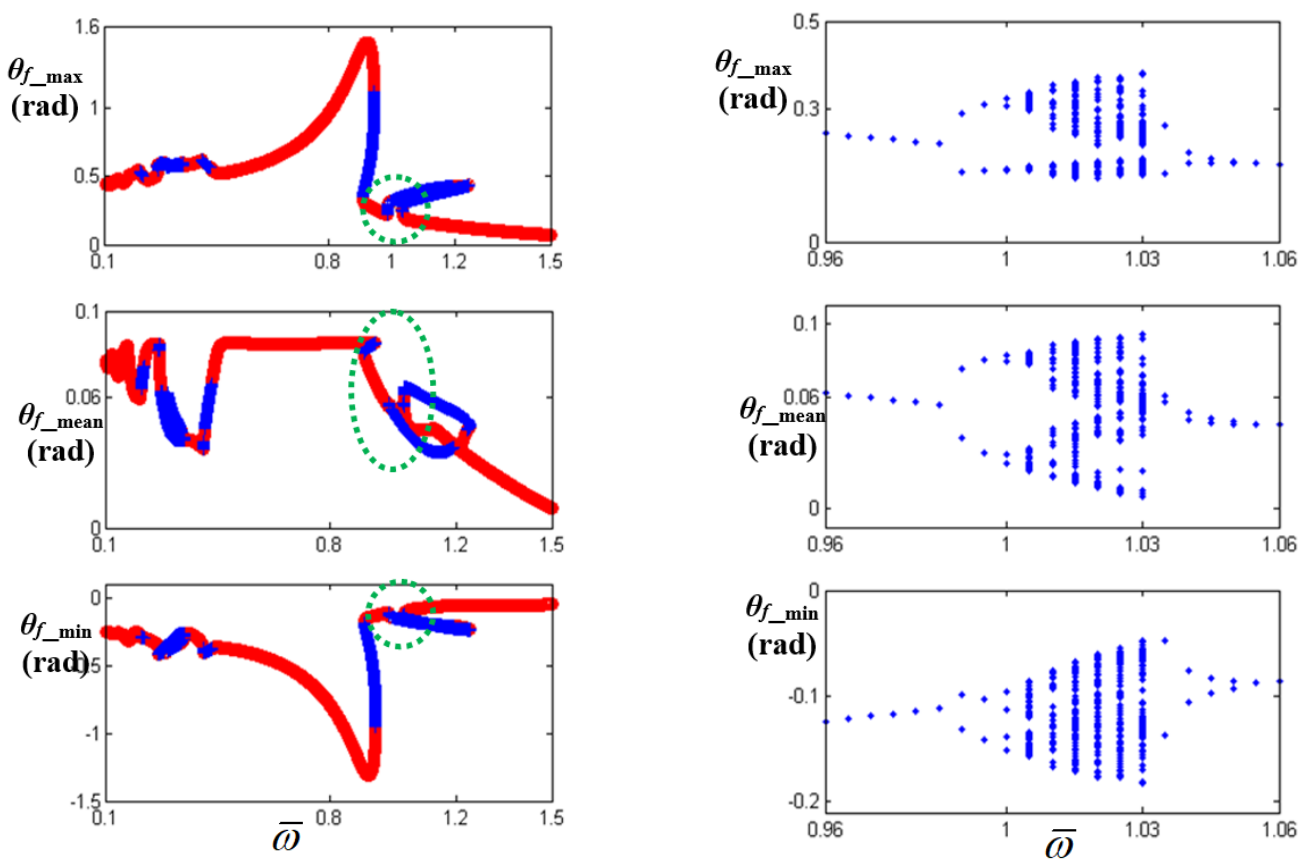

(a)

(b)

Figure 9. Stability conditions from HBM with max, mean and min values, and its bifurcation diagrams at the sub-harmonic region: (a) stable and unstable conditions in a frequency response with max, mean and min values; (b) bifurcation diagram of the sub-harmonic responses. Key: $\mathbf{O}$, stable solutions of $\mathrm{HBM} ;+$, unstable solutions of $\mathrm{HBM} ; \bullet$, numerical results of the bifurcation. 


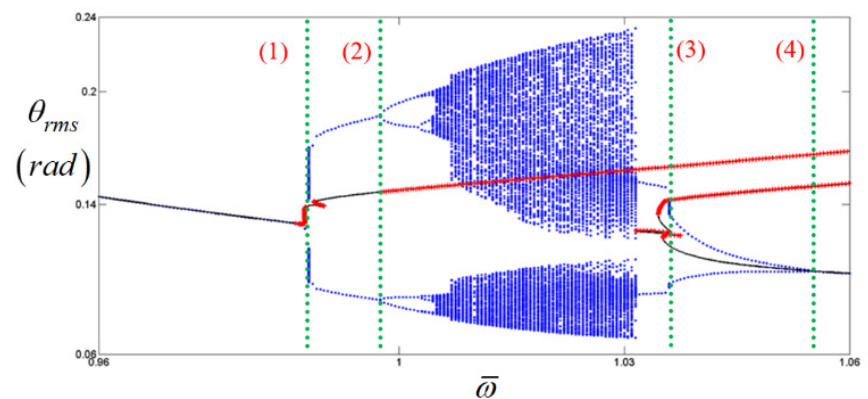

Figure 10. Bifurcation diagram projected in the sub-harmonic region calculated by HBM with RMS. Key: — , stable solutions of $\mathrm{HBM} ;+$, unstable solutions of HBM; $\bullet$, numerical results of the bifurcations.
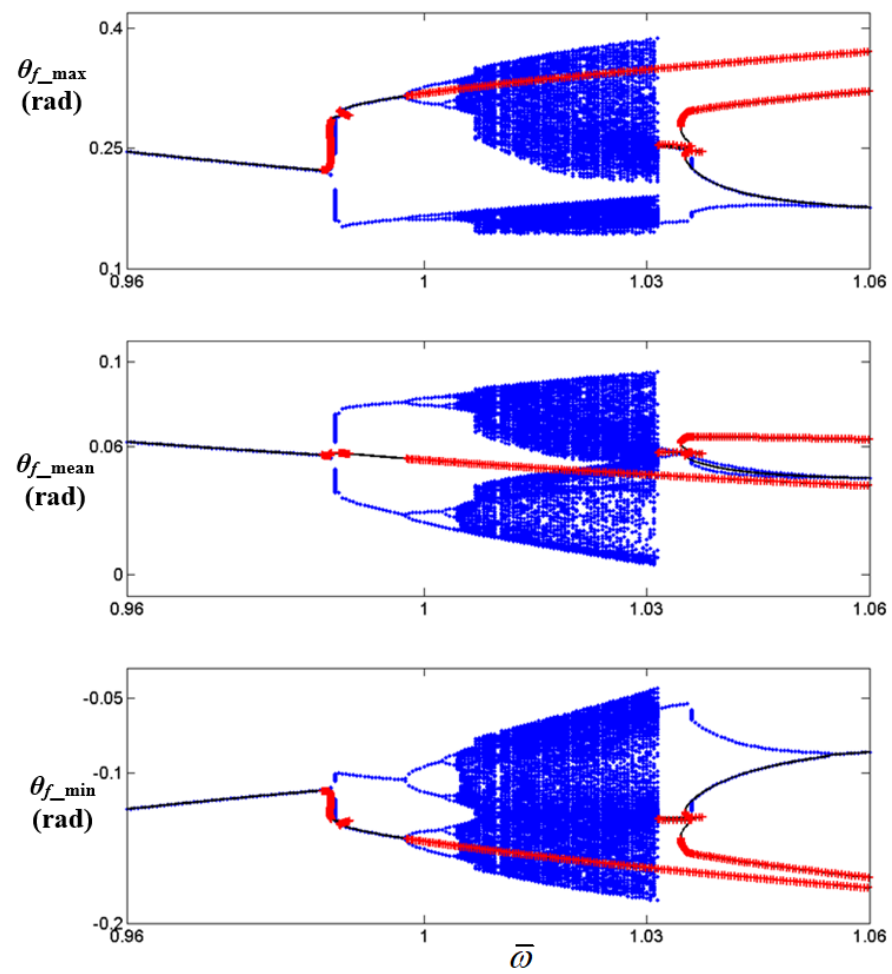

Figure 11. Bifurcation diagrams projected in the sub-harmonic region calculated by HBM with max, mean and min values. Key: —_, stable solutions of HBM; +, unstable solutions of HBM; numerical results of the bifurcations.

\section{Conclusions}

This study examined nonlinear dynamic characteristics by focusing on sub-harmonic regimes. To examine the nonlinear system responses, both the HBM and NS were employed, and their results were compared. For example, the overall system responses were examined based on the HBM by including the super- and sub-harmonic responses. In order to reveal the sub-harmonic responses, the additional numerical techniques were implemented. Then, the stable and unstable responses have been investigated by comparing the NS results which could lead us to understanding the relationships of stability conditions to the bifurcations well. For the contributions of this article, we first investigated the sub-harmonic responses which are determined as unstable conditions based on the HBM. In addition, numerical techniques have been suggested to reveal sub-harmonic responses regarding HBM. Second, to understand the nonlinear dynamic behaviors which are normally determined as unstable conditions, bifurcation analysis was implemented and compared with the frequency responses by focusing on the sub-harmonic regimes. This could result in an 
improved understanding of the nonlinear dynamic behaviors of a practical system and suggest a complementary analysis with both HBM and NS.

This work was conducted to examine mostly the sub-harmonic effects with respect to torsion vibratory motions. However, there are still various deviations and complex behaviors in the course of frequency sweeping conditions affected by super-harmonics. In addition, the various conditions for the broader range of sub-harmonic responses will be investigated as for the next stage of research.

Author Contributions: J.-Y.Y. and B.K. initiated and developed the ideas related to this research work. J.-Y.Y. and B.K. developed novel methods, derived relevant formulations, and carried out performance analyses and numerical analyses. J.-Y.Y. wrote the paper draft under B.K.'s guidance and B.K. finalized the paper. All authors have read and agreed to the published version of the manuscript.

Funding: This work was supported by an Incheon National University Research Grant in 2018 (20180019).

Institutional Review Board Statement: Not applicable.

Informed Consent Statement: Not applicable.

Data Availability Statement: Not applicable.

Conflicts of Interest: The authors declare no conflict of interest.

\section{References}

1. Yoon, J.Y.; Singh, R. Effect of multi-staged clutch damper characteristics on transmission gear rattle under two engine conditions. Proc. IMechE Part D J. Automob. Eng. 2013, 227, 1273-1294. [CrossRef]

2. Yoon, J.Y.; Yoon, H.S. Nonlinear frequency response analysis of a multistage clutch damper with multiple nonlinearities. ASME J. Comput. Nonlinear Dyn. 2014, 9, 031007. [CrossRef]

3. Peng, Z.K.; Lang, Z.Q.; Bilings, S.A.; Tomlinson, G.R. Comparison between harmonic balance and nonlinear output frequency response function in nonlinear system analysis. J. Sound Vib. 2008, 311, 56-73. [CrossRef]

4. Chen, Y.M.; Liu, J.K.; Meng, G. Incremental harmonic balance method for nonlinear flutter of an airfoil with uncertain-butbounded parameters. Appl. Math. Model. 2012, 36, 657-667. [CrossRef]

5. Al-shyyab, A.; Kahraman, A. Non-linear dynamic analysis of a multi-mesh gear train using multi-term harmonic balance method: Sub-harmonic motions. J. Sound Vib. 2005, 279, 417-451. [CrossRef]

6. Masiani, R.; Capecchi, D.; Vestroni, F. Resonant and coupled response of hysteretic two-degree-of-freedom systems using harmonic balance method. Int. J. Non-Linear Mech. 2002, 37, 1421-1434. [CrossRef]

7. Raghothama, A.; Narayanan, S. Bifurcation and chaos in geared rotor bearing system by incremental harmonic balance method. $J$. Sound Vib. 1999, 226, 469-492. [CrossRef]

8. Raghothama, A.; Narayanan, S. Bifurcation and chaos of an articulated loading platform with piecewise non-linear stiffness using the incremental harmonic balance method. Ocean Eng. 2000, 27, 1087-1107. [CrossRef]

9. Yang, Y.S.; Liu, X. Nonlinear dynamics of a spur gear pair with time-varying stiffness and backlash based on incremental harmonic balance method. Int. J. Mech. Sci. 2006, 48, 1256-1263.

10. Duan, C.; Rook, T.E.; Singh, R. Sub-harmonic resonance in a nearly pre-loaded mechanical oscillator. Nonlinear Dyn. 2007, 50, 639-650. [CrossRef]

11. Wong, C.W.; Zhang, W.S.; Lau, S.L. Periodic forced vibration of unsymmetrical piecewise-linear systems by incremental harmonic balance method. J. Sound Vib. 2006, 48, 1256-1263. [CrossRef]

12. Kim, T.C.; Rook, T.E.; Singh, R. Super-And sub-harmonic response calculation for a torsional system with clearance nonlinearity using the harmonic balance method. J. Sound Vib. 2005, 281, 965-993. [CrossRef]

13. Miguel, L.P.; Teloli, R.O.; Silva, S. Some practical regards on the application of the harmonic balance method for hysteresis models. Mech. Syst. Signal Process. 2020, 143, 106842. [CrossRef]

14. Detroux, T.; Renson, L.; Masset, L.; Kerschen, G. The harmonic balance method for bifurcation analysis of large-scale nonlinear mechanical systems. Comput. Methods Appl. Mech. Eng. 2015, 296, 18-38. [CrossRef]

15. Xie, L.; Baguet, S.; Prabel, B.; Dufour, R. Bifurcation tracking by Harmonic Balance Method for performance tuning of nonlinear dynamical systems. Mech. Syst. Signal Process. 2017, 88, 445-461. [CrossRef]

16. Von Groll, G.; Ewins, D.J. The harmonic balance method with arc-length continuation in rotor/stator contact problems. J. Sound Vib. 2001, 241, 223-233. [CrossRef]

17. Deconinck, B.; Nathan Kutz, J. Computing spectra of linear operators using the Floquet-Fourier-Hill method. J. Comput. Phys. 2006, 219, 296-321. [CrossRef]

18. Lei, H.; Huizheng, C.; Yushu, C.; Kuan, L.; Zhansheng, L. Bifurcation and stability analysis of a nonlinear rotor system subjected to constant excitation and rub-impact. Mech. Syst. Signal Process. 2019, 125, 65-78. 
19. Lei, H.; Yushu, C. Analysis of $1 / 2$ sub-harmonic resonance in a maneuvering rotor system. Sci. China Technol. Sci. 2014, 57, 203-209.

20. Yoon, J.Y.; Byeongil, K. Stability and bifurcation analysis of super- and sub-harmonic responses in a torsional system with piecewise-type nonlinearities. Sci. Rep. 2021, 11, 1-18. [CrossRef]

21. Dormand, J.R.; Prince, P.J. A family of embedded Runge-Kutta formulae. J. Comput. Appl. Math. 1980, 6, 19-26. [CrossRef]

22. Seydel, R. Practical Bifurcation and Stability Analysis. Springer: Berlin/Heidelberg, Germany, 1994. 